\title{
Percepción de Estudiantes de Psicología sobre el Uso de Facebook para Desarrollar Pensamiento Crítico
}

\author{
Pablo J. Castro e Ingrid N. González-Palta \\ Universidad de La Serena, Departamento de Psicología, Matta №147, Coquimbo-Chile. \\ (e-mail: pablocastro@userena.cl; igonzalez@userena.cl)
}

Recibido Jul. 15, 2015; Aceptado Sep. 7, 2015; Versión final Oct. 2, 2015, Publicado Feb. 2016

\begin{abstract}
Resumen
El objetivo del presente trabajo es reportar una experiencia de implementación de un grupo en Facebook como apoyo al desarrollo del pensamiento crítico en alumnos de una asignatura de la carrera de psicología de la Universidad de La Serena en Chile. Se realizó una encuesta anónima y voluntaria a 43 alumnos al finalizar el curso. El análisis de los resultados muestra que la mayoría de los estudiantes perciben que el uso de esta estrategia y metodología empleada contribuyeron al desarrollo del pensamiento crítico. Se observa un grado general de satisfacción y una actitud favorable a la incorporación de esta plataforma como complemento a las clases. Se concluye que el uso de grupos en Facebook puede ser una estrategia útil para desarrollar pensamiento crítico en la formación universitaria.
\end{abstract}

Palabras clave: plataformas virtuales, Facebook, pensamiento crítico, enseñanza de la psicología, redes sociales

\section{Psychology Students' Perceptions about using Facebook to Develop Critical Thinking}

\begin{abstract}
The objective of this work is to report the experience of implementing a Facebook group as support to the development of critical thinking of the students of the psychology program of Universidad de La Serena in Chile. An anonymous and voluntary survey was applied to 43 students after finishing the course. The data analysis shows that the majority of the students perceive the use of this strategy and methodology as an initiative that contributed to the development of critical thinking. A general degree of satisfaction and positive attitude towards the inclusion of this platform as complement to classes are observed. It is concluded that the use of Facebook groups can be a useful tool to develop critical thinking in university education.
\end{abstract}

Keywords: virtual platforms, Facebook, critical thinking, teaching of psychology, social networking 


\section{INTRODUCCIÓN}

La actual llamada sociedad del conocimiento/información en la que estamos insertos es altamente competitiva, dinámica, variable y compleja (González et al., 2010; Rodríguez-Ponce, 2010). Caracterizada, entre otros aspectos, por el rápido incremento en la producción de conocimiento, los procesos de innovación y el uso de nuevas tecnologías (David y Foray, 2002 citado en Micheli y Armendáriz, 2011). Esta celeridad también la observamos en el mundo laboral, que en continuo cambio, demanda a las universidades la formación de profesionales autónomos, que actualicen permanentemente conocimientos y competencias para hacer frente a las necesidades, también dinámicas, de la sociedad (Álvarez et al., 2008; Fernández y Bernardo, 2011; Lamas, 2008).

En este marco se han propiciado consensos educativos respecto a la necesidad de formar estudiantes que sean activos (Peñalosa et al., 2006) y críticos en los procesos de construcción de su conocimiento. Lo anterior llamado a veces también como el centramiento en el aprendizaje más que en la enseñanza ha devenido en estrategias educativas interesadas en que los estudiantes elaboren lo aprendido, esta elaboración puede considerar procesos de crítica al conocimiento presentado por el docente o por los autores revisados en una asignatura o a sus contextos de aplicación. En este mismo sentido, Moreno y Quiñones (2009) señalan que el proceso de aprendizaje ocurre cuando aquello que se aprende es "objeto de elaboración personal y consciente, a través del procesamiento cognitivo y vivencial” ( p. 5).

Una de las habilidades consideradas prioritarias en diversos currículos de educación media y superior ha sido la denominada "pensamiento crítico". Cabe mencionar que existen diferentes definiciones y abordajes del constructo (Moore, 2013). Los elementos comunes a estas diversas definiciones de pensamiento crítico en el contexto de la educación superior, apuntan a considerar que es una habilidad que posee un sujeto inquisitivo consistente en: identificar ideas centrales y supuestos de un argumento; reconocer relaciones importantes, hacer inferencias correctas a partir de datos; deducir conclusiones desde la información; evaluar evidencias y autoridad del autor o fuente de un argumento. Junto con lo anterior, alguien que ha desarrollado pensamiento crítico poseería la competencia para evaluar su propio pensamiento y el de los demás. A nivel de disposición, se entiende que tener pensamiento crítico conlleva actitudes de empatía, humildad, perseverancia, rectitud, prudencia, mentalidad abierta, entre otras (Wass, Harland y Mercer, 2011).

Se ha determinado que existen tres principales enfoques sobre pensamiento crítico: filosófico, pedagógico y psicológico, concluyéndose que lo común en ellos es considerar que el pensamiento crítico se caracteriza por una disposición a la reflexión y conocimiento contextual. Una definición de la actividad de pensar críticamente y que ha intentado integrar los mencionados enfoques, es la propuesta reciente de Hammer y Noemi (en prensa), quienes la entienden como "la acción de enjuiciar deliberadamente el conocimiento disponible el que, al registrarse simbólicamente en un código lingüístico, se expresa en la forma de discurso, por lo que el análisis de contenido permite evaluar, al menos parcialmente, la calidad de aquél en cuanto competencia" (p. 5).

Situándonos ahora en el caso de la enseñanza de la psicología en Chile, en los últimos años ha surgido la necesidad de actualizar los currículum y los perfiles de egreso en la formación terciaria. Con este fin, desde el año 2007, la Red de Psicología del CUECH ha consensuado competencias profesionales y genéricas o transversales de formación para el perfil de egreso de los profesionales psicólogos. Una de las competencias genéricas es el pensamiento crítico (Davies, 2011). Esta competencia alude a la capacidad de "evaluar y discriminar reflexivamente sobre diferentes fenómenos del ámbito personal, académico, profesional y del cotidiano, con una mirada prospectiva, compleja y global, orientado a la acción" (Red Psicología Proyecto MECESUP ULS 0601, 2013, p. 179).

Desarrollar la competencia de pensamiento crítico en los psicólogos en formación importa y por muchas razones. Siguiendo las ideas expuestas por Betancourth et al., (2012) desarrollar el pensamiento crítico en pregrado es crucial para favorecer características inquisitivas, flexibles, honestas, y justas en relación a su razonamiento y cognición por parte del alumno. De igual modo, en psicología tendría relevancia debido a la complejidad y multicausalidad de los fenómenos sociales, que interpelan al profesional a examinar, comprender y tomar decisiones, entre muchas alternativas, de la mejor forma de analizar e intervenir con una persona, grupo o comunidad, haciéndose cargo de sus limitaciones profesionales y de su propia forma de pensar (Machado, 2014). Al respecto, en las reflexiones surgidas al interior de la profesión, se visualiza al profesional psicólogo como un sujeto con compromiso social, asumiendo para ello un rol que propicie la transformación de su sociedad y el aporte al conocimiento, esto desde "una postura más crítica frente a lo que sucede en la sociedad" (Colegio de psicólogos de Chile, 1999; Zuñiga, 2013, p. 96). 
Así mismo, se ha observado la relación del pensamiento crítico con el rol profesional ya que es su mirada particular y comprensiva de los fenómenos lo que caracteriza y distingue al psicólogo en el mundo laboral (González et al., 2014), probablemente guarde relación con el currículum de enseñanza que supone un psicólogo ideal donde las habilidades de análisis y comprensión son fundamentales (Cabrera et al., 2010). En este mismo contexto los psicólogos egresados observan mayores dificultades en las competencias genéricas o transversales, más que las específicas (González et al., 2014), importancia que coincide además con lo percibido por formadores de psicólogos y estudiantes (Cabrera et al., 2010). Lo anterior es coincidente con los hallazgos de un estudio realizado con formadores de psicólogos en Estados Unidos y Canadá, observándose que el objetivo de enseñanza que presenta mayor coincidencia entre los docentes es el avance del pensamiento crítico en sus estudiantes (Domenech et al., 2014). En suma, pareciera ser que desarrollar el pensamiento crítico en los estudiantes sería una tarea prioritaria en la formación de psicólogos en tanto que favorece un ejercicio de la profesión no aséptico sino que situado en contexto.

\section{TIC's y Redes Sociales como medios educativos}

En las últimas décadas las Tecnologías de la Información y la Comunicación se han utilizado como una de las estrategias educativas que buscan propiciar la competencia mencionada previamente. Esta situación se evidencia en la proliferación de investigaciones en los últimos años respecto de la efectividad de las plataformas de mediación pedagógica (e-learning, b-learning, campus virtuales, etc). Una de las más conocidas es Moodle, plataforma gratuita que ya en el año 2011 se utilizaba en más de 200 países. Otras investigaciones evidencian los alcances y limitaciones del uso de estas tecnologías en la educación (Abarca, 2013; Aguirre y Manasía, 2009; Bosch, 2009; González, 2012). Al respecto, debemos mencionar que la utilización de estas aplicaciones "no son un fin en sí mismas sino un medio para resolver problemas y modificar los escenarios futuros" (Chan, 2005 citado en Muñoz-Cano et al., 2012, p.4). A este respecto, se infiere que su sola inclusión no asegura un mejor aprendizaje en el alumnado, no obstante su uso sí supondría la integración de nuevos entornos y metodología, que pudiese convertirse en un valioso apoyo para la labor docente y por sobre todo para el aprendizaje del estudiante.

Diversos autores muestran que el uso de las redes sociales fomentaría la autonomía en el aprendizaje, la motivación (intrínseca y extrínseca), y el trabajo colaborativo entre pares (Bosch, 2009; González, 2012). Así, pareciera ser que las características de este tipo de espacios como el ser abiertos, conocidos, de fácil acceso y horizontales favorece que el alumno se convierta en un "agente autónomo, participativo y generador de ideas, contenidos y aprendizaje” (González, 2012 , p. 40). Lo anterior apoya una de las premisas de esta investigación, a saber, la idea de conquistar nuevos espacios de aprendizaje e "Ir" donde están los alumnos más que esperar que vengan ellos a los espacios planteados desde fuera (González, 2012).

\section{Características de Face-Crític y de su implementación}

La implementación de la que se da cuenta en este trabajo, consideró como contexto aspectos del modelo educativo de la institución universitaria. Dicho modelo busca desarrollar en los estudiantes de todo programa de formación de la institución la competencia de dominio de las tecnologías de la comunicación y la información, la autonomía del estudiante y el logro de una visión crítica de su entorno. Por lo que la implementación de la que aquí se da cuenta se consideró como un aporte al logro de estas competencias genéricas que este modelo declara como sello de formación (Universidad de La Serena, 2011). Con el objetivo de desarrollar las competencias mencionadas se creó un grupo en Facebook llamado "FaceCrític", que era parte formal de la asignatura "teorías de la personalidad". Su incorporación fue voluntaria, y se inscribieron 52 miembros (100\% de los alumnos del curso).

La siguiente fue la descripción del grupo en Facebook disponible para sus miembros: "Espacio virtual de la asignatura Teorías de la Personalidad año 2014 de la carrera de Psicología de la ULS. El objetivo es complementar el debate de las clases por este medio como apoyo al desarrollo del pensamiento crítico. Todos los alumnos de la asignatura pueden publicar y estimular la discusión por esta vía. Este grupo se encuentra en el marco del proyecto "Face-Crític: Favoreciendo el pensamiento crítico y el aprendizaje autónomo a través de un espacio virtual." que elaboramos y que resultó seleccionado para ser financiado por la dirección de Docencia de la ULS a través de su concurso de buenas prácticas docentes 2014. Saludos y bienvenidos al curso." Una presentación más extensa se realizó presencialmente en la primera clase de la asignatura. En la figura 1 se puede observar una imagen del grupo Face-Crític.

Face-Crític consistía en la presentación de diversos estímulos de discusión relacionados a los contenidos tratados en las clases presenciales de la asignatura Teorías de la personalidad. Los estímulos fueron textos múltiples (preguntas abiertas, noticias, artículos, videos, etc.) subidos uno por semana, por el 
docente, la psicóloga de apoyo al proyecto o espontáneamente por los alumnos. A partir de estos estímulos los estudiantes elaboraron comentarios argumentados. Cada semana se elegía los mejores comentarios, quienes obtenían una bonificación para la instancia evaluativa del curso.

El presente reporte tiene como objetivo dar a conocer la percepción que tienen los estudiantes de segundo año de la carrera de psicología de la Universidad de La Serena acerca del uso de Facebook para el desarrollo del pensamiento.

\section{METODOLOGÍA}

Se detalla la metodología en tres apartados principales: población usada, como se hizo la implementación y cómo se realizó el proceso evaluación de face-crític

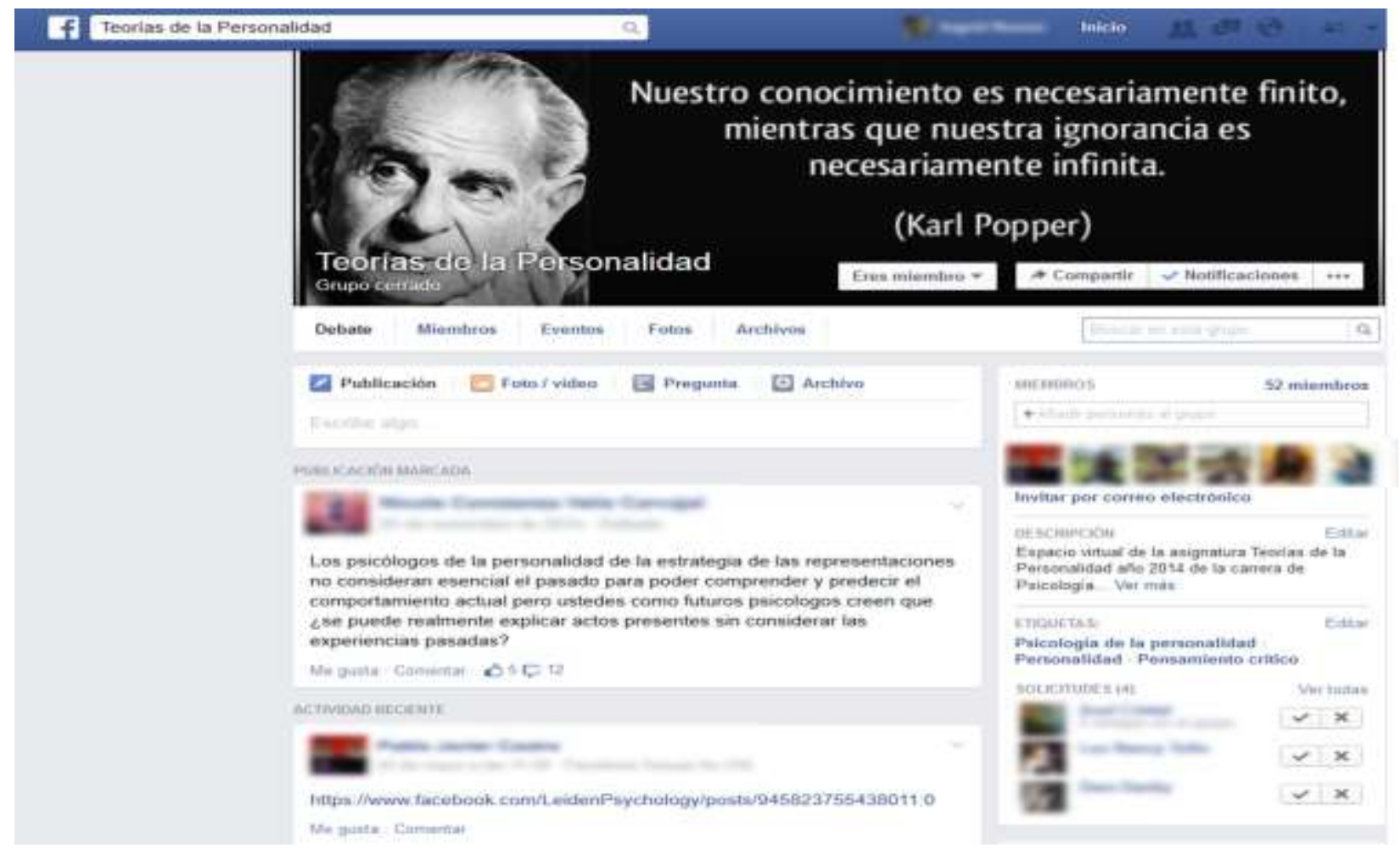

Fig. 1: Imagen del grupo Facebook utilizado en la asignatura Teorías de la Personalidad.

\section{Población}

De los 51 alumnos inscritos en la plataforma Face-Crític, 43 contestaron la encuesta de evaluación voluntaria, lo que corresponde al $84 \%$ de la población. De estos participantes 33 fueron mujeres y 10 hombres.

\section{Implementación}

La plataforma Face-Crític se implementó durante los meses de Agosto a Diciembre, correspondiente al $2^{\circ}$ Semestre académico del año 2014.

\section{Evaluación de Face-Crític}

El proceso de evaluación fue a través de una encuesta anónima y voluntaria a los estudiantes al finalizar el curso. Con lo anterior se conoció la percepción sobre el aporte que realiza Face-Crític al desarrollo del pensamiento crítico asociado al nivel de autonomía en el aprendizaje, y el grado general de satisfacción de los alumnos, respecto de la plataforma. La encuesta que fue diseñada por los autores estaba dividida en tres secciones, la primera recoge información sociodemográfica general, la segunda: veinticinco reactivos de respuesta sí/no, y la tercera siete preguntas de alternativas.

En este trabajo se describen los resultados referidos exclusivamente a las dimensiones que son el objeto del presente trabajo: Pensamiento crítico asociado a la autonomía en el aprendizaje que pudiera haber generado el uso de esta herramienta virtual. Posteriormente se realizó un análisis de frecuencia de los 
datos utilizando el software Microsoft Excel. Junto a lo anterior, en la plataforma Face-Crític espontáneamente surgió de parte de los alumnos un proceso de evaluación de la misma, por lo que se presentan aquí algunas citas textuales que ejemplifican y enriquecen cualitativamente los datos de percepción recogidos por la encuesta.

\section{RESULTADOS Y DISCUSIÓN}

\section{Pensamiento crítico y aprendizaje}

En la tabla 1 se muestra la valoración que realizan los alumnos sobre la contribución de Face-Crític al desarrollo del pensamiento crítico y al aprendizaje de los contenidos de la asignatura "Teorías de la Personalidad". Esta tabla da cuenta de algunas limitaciones y beneficios percibidos por los estudiantes luego de la utilización de Face-Crític como metodología de enseñanza-aprendizaje.

Tabla 1: Resultado sobre pensamiento crítico y aprendizaje.

\begin{tabular}{|l|l|l|}
\hline Indicador & Sí & No \\
\hline Creo que Face-Crític contribuye al desarrollo del pensamiento crítico & $88 \%$ & $12 \%$ \\
\hline $\begin{array}{l}\text { Un estímulo o comentario de mi compañero me ayudó a tener una mirada diferente sobre } \\
\text { un tema }\end{array}$ & $67 \%$ & $33 \%$ \\
\hline Me gustó tener un espacio paralelo a las clases para pensar en los temas de personalidad & $72 \%$ & $28 \%$ \\
\hline
\end{tabular}

Un beneficio percibido, por la mayoría de los estudiantes, es que este espacio virtual contribuyó a pensar críticamente los distintos enfoques, metodologías y supuestos que están a la base de la psicología de la personalidad. Si bien, este estudio no cuenta con una medición objetiva del pensamiento crítico, sí es relevante el que subjetivamente los alumnos perciban una mejora en dicho proceso, ya que, se instala en ellos la relevancia que tiene el pensar críticamente para el ejercicio profesional y estudio de la psicología. Como se ha mencionado anteriormente, la de Psicología del CUECH ha determinado competencias profesionales y transversales de formación para el perfil de egreso de los profesionales psicólogos, y tanto los docentes como los egresados (Domenech et al., 2014; González et al., 2014). Competencias que al salir al mundo laboral, son percibidas por los egresados como de importancia para su quehacer, entre ellas la de pensamiento crítico. Por lo anterior, es pertinente que ya desde el pregrado se comience a socializar e instalar en los alumnos la relevancia que tiene perfeccionar este tipo de pensamiento desde su formación. Así lo argumenta un alumno en el contexto de Face-Crític: Creo (...) que la idea de generar pensamiento crítico es espléndida. Siento que es el pensamiento crítico el que nos permite evolucionar tanto personal como social y culturalmente. Nos aleja del conformismo, nos 'abre los ojos', en otras palabras nos da la posibilidad de ser entes únicos y no simples borregos (Estudiante 1).

Es relevante observar que un $67 \%$ de los alumnos consideran que la visión entregada por un par le permitió considerar un punto de vista distinto del argumentado por él (Tabla 1). Este resultado parece influido por una de las contribuciones que hace la utilización de Face- Crític, la de facilitar la interacción entre pares y entre profesor y alumnos. En esta plataforma virtual es posible comunicarse de manera abierta y flexible, espacio que no siempre es posible generar en la sala de clases, donde, por ejemplo, muchos alumnos no se sienten con la libertad de expresar todas sus opiniones (este punto se discutirá más tarde). Desde esta perspectiva, el contar con espacios que favorezcan el encuentro de opiniones y de reflexión en un grupo virtual, permitiría enriquecer el aprendizaje y la construcción de nuevas ideas sobre un determinado tema (Paul y Elder, 2005).

El resultado expuesto, da cuenta de la relevancia del proceso dialógico y de discusión en el desarrollo del pensamiento crítico, es decir, encontrarse en la conversación con posturas distintas permitirán relativizar sus propias ideas, o tomar conciencia sobre ideas alternativas. Relacionado a lo anterior una alumna señala respecto de Face- Crític: Cuando surge la discusión se vuelve en una buena instancia para aprender más sobre el tema si no hubiese sido quizás por este espacio yo no me hubiese motivado a indagar mucho más sobre los autores y las críticas de sus teorías. Yo creo que el hecho de ponernos en aprietos rebatiendo muchas veces o tratan de indagar más allá es lo que más rescato de este espacio (Estudiante 2).

Los alumnos (78\%) consideran como positivo el hecho de contar con un espacio extra aula para reflexionar sobre los temas tratados en clases (Tabla 1). Ellos perciben que este espacio contribuye a la 
comprensión y aplicación de los supuestos, conceptos, hipótesis e implicancias metodológicas de los principales enfoques teóricos de la personalidad. Este es un resultado de aprendizaje relevante del programa de estudio de la asignatura. Este resultado se contrapone con la investigación desarrollada por Muñoz-Cano et al., (2012), donde los estudiantes no mostraron aceptación a una plataforma b-learning, en particular una disposición negativa hacia los espacios donde debían elaborar escritos y participar en foros.

El contar con este espacio virtual se relaciona con las posibilidades que abre el uso de herramientas virtuales en el aprendizaje, es decir, la opción de interactuar sincrónica y asincrónicamente entre profesor y alumno superando límites temporales y espaciales (Abarca, 2013). Al respecto una de las alumnas señala: Estoy segura que este proyecto quizás no les cambie el pensamiento a todos, pero nos hizo reflexionar, darle una vuelta más al asunto, quizás algunos por tiempo no pudimos estar tan metidos, pero (...) todos lo que aquí comentaban le estaban dando una vuelta más a la teoría, y así mismo la interiorizaban y la hacían propia, criticándola, queriendo cambiarla, desechándola, contrastándola, todos tenemos un momento en nuestra vida que algo nos hace clic, nos cambia los esquemas, y quizás alguno sí le hizo sentido esto puede ayudar ¿Por qué no? a mi gusto el fomentar esta instancia hizo que aflorara en cada uno de los comentarios un pensamiento crítico (Estudiante 3). La alumna hace referencia a cómo los estímulos publicados en Face-crític, podrían contribuir al desequilibrio cognitivo y/o al aprendizaje por insight como elementos del cambio de esquemas de pensamiento (Manterola, 2003).

Es importante considerar que el beneficio que trae la utilización de Facebook de superar las barreras de tiempo y espacio de la enseñanza en el aula, entregando posibilidades extra aula, viene aparejada con la necesidad de realizar una mayor inversión de tiempo por parte del docente (Bravo, 2012), ya que para hacer efectiva la interacción tendrá que responder preferentemente de manera contingente a los comentarios de los estudiantes y diseñar o seleccionar estímulos adecuados. Sin embargo, existe en muchas ocasiones poco uso por parte de los alumnos de los horarios de atención de alumnos que ofrecen los docentes, y podría utilizarse este tiempo disponible del profesor para retroalimentar a los estudiantes en esta plataforma.

\section{Actitud de los alumnos hacia Face-Crític}

En la tabla 2 se muestran los resultados relacionados con la actitud que los estudiantes desarrollaron ante el uso de esta plataforma. Destaca entre estos resultados que un $67 \%$ de los encuestados afirma que a pesar de no escribir comentarios en el grupo Facebook, sí participaban de él revisando lo aportado por sus compañeros. Se han estudiado dos patrones o tipos de actividad en el uso de Facebook en adultos y adolescentes: un uso pasivo o de "consumo", como por ejemplo leer lo que otros publican, y la comunicación dirigida (Burke et al., 2010; Shaw, 2015). En el caso de las plataformas virtuales creadas con fines de aprendizaje, la participación pasiva tiende a ser mayoritaria cuando la utilización de la plataforma es opcional y complementaria a la docencia presencial, presentándose tasas de participación activa cercanas a un tercio de los estudiantes (Jia et al., 2012), las que sí tienden a ser mayores cuando se compara Facebook con otras plataformas como los blogs o Moodle, ya que en estas últimas se reportan porcentajes mayores de usuarios pasivos (González, 2012). En nuestro caso, y si consideramos que participar, incluye también el leer y reflexionar o acomodar/asimilar conocimiento a partir de lo expuesto por compañeros, podemos concluir que se logró una participación mayoritaria de los alumnos en Face-Crític.

Tabla 2: Resultados de la actitud de los alumnos hacia Face-Crític

\begin{tabular}{|l|l|l|l|}
\hline Indicador & Sí & No & No contesta \\
\hline $\begin{array}{l}\text { A pesar de no comentar observaba activamente la participación de } \\
\text { mis compañeros en el grupo Face-Crític }\end{array}$ & $67 \%$ & $33 \%$ & \\
\hline Me toma mucho tiempo comentar en Facebook & $35 \%$ & $53 \%$ & $12 \%$ \\
\hline Me da verguenza que los demás lean mis comentarios & $26 \%$ & $65 \%$ & $9 \%$ \\
\hline
\end{tabular}

Resulta interesante también que varios estudiantes (53\%) descartan la afirmación que hace referencia a que el uso de Face-Crític les demandaría mucho tiempo (versus un $35 \%$ que indica que sí). Esto contrasta con otras hipótesis que plantean que una limitante de estas plataformas virtuales es la demanda de tiempo extra que implica para el estudiante (Inzunza et al., 2012), aun cuando un tercio de los estudiantes de esta experiencia indicaron que la falta de tiempo sí era una causa de no participación, al respecto una alumna, que participaba activamente en el grupo, opinó: Quizás algunos por tiempo no pudimos estar tan metidos (Estudiante 3). Lo anterior nos hace pensar en que se requiere de más indagaciones que ponderen con 
certeza si el factor tiempo es o no una limitante para el uso de plataformas virtuales, tanto para los estudiantes como para los docentes. Lo anterior considerando el hecho de que es posible que en algunas experiencias los estudiantes argumenten la falta de tiempo como una causal de no participación, cubriendo bajo esa justificación otras limitantes o razones para su no usar la platafoma.

Relacionado también a la participación de los estudiantes en el grupo Facebook, surgió una dinámica recurrente en la implementación de la experiencia descrita aquí, un grupo de alumnas comenzó a participar activamente en la plataforma con comentarios de buen nivel de elaboración, por el contrario en la sala de clase ocurrió que eran mayoritariamente varones quienes participaban activamente de las discusiones y las estudiantes, salvo excepciones, tenían una participación más pasiva. Este hecho puede ser interpretado desde diversas perspectivas, alguna de las cuales escapan a las posibilidades de este reporte de experiencia, sin embargo es posible hipotetizar, apoyados en literatura respecto al uso de Facebook por estudiantes universitarios (Bosch, 2009), que las estudiantes podrían haber percibido el espacio de Facebook como más privado que la sala de clases. Otras posibles hipótesis podrían ir por el lado de; diferencias de género en relación a la disposición a argumentar cara a cara y su función de competitividad o de búsqueda de beneficios individuales (Hample, 2005; Hample et al., 2010), tipo de motivación predominante (extrínseca, intrínseca) según el género (por ejemplo, mayor o menor interés en las calificaciones o en el aprendizaje). Finalmente, cabe recordar que en el contexto de los que se denomina las ecologías argumentativas los "hablantes naturales consideran que sus acciones argumentativas están sujetas a ciertos parámetros normativos" (Santibañez, 2014, p. 178) y por tanto otros factores culturales y de contexto incidirán en la participación mayor o menor en la sala de clases o en una plataforma virtual.

Respecto de la exposición social que genera publicar algo en el grupo, es decir, la situación de que los demás participantes de él pueden leer y juzgar lo escrito por cualquiera de ellos, se preguntó en la encuesta si la vergüenza podría haber sido un inhibidor. Ante ello un $65 \%$ de los estudiantes indicó que esto no fue así. Esto es coherente con estudios que relacionan el uso de Facebook con la ansiedad social (v.g., Shaw, 2015), es decir, la vergüenza podría ser un factor un inhibidor de la participación cara a cara en la sala de clases más que en esta plataforma.

En síntesis, según los resultados anteriores sobre la actitud hacia Face-Crític, se confirma lo señalado por otras investigaciones, en cuanto a una disposición favorable a participar de nuevas experiencias didácticas que utilicen plataformas virtuales (Çelik, 2010; Paragina et al., 2011; Sánchez et al., 2009; Sava et al., 2010; Young y Norgard, 2006).

\section{Evaluación de Face-Crític}

Uno de los beneficios de incorporar estrategias virtuales en los procesos de aprendizaje tiene que ver con la posibilidad de incorporar "textos múltiples" (Nokes et al., 2007), es decir, la facilidad para presentar distintos estímulos, por ejemplo, el uso de videos, audios, citas, imágenes, etc. Face-Crític incorporó como estímulos de discusión diversos elementos, como los mencionados anteriormente, por lo que interesaba saber cuál de todos ellos era el que más atraía y favorecía la movilización de la reflexión y del pensamiento crítico en los estudiantes. Según la percepción de los alumnos los videos, seguido de las citas y las fotografías fueron los más atractivos (Tabla 3). Utilizar esta modalidad de estímulos de discusión es cada vez más relevante con las nuevas generaciones de estudiantes que han estado expuestos desde su infancia a tecnologías inteligentes (celulares, tablets, etc).

Resultaría interesante conocer qué otras características deben tener los estímulos propuestos por los docentes para favorecer el pensamiento crítico, más allá del tipo de texto que se utilice, y si éstas características son distintas si se dan en una interacción cara a cara con los alumnos versus plataformas virtuales como Facebook o Moodle.

Otra posibilidad de mejora que permite Face-Crític es el de juntar en un mismo espacio virtual alumnos de distintos cohortes, lo que generalmente es complejo de realizar en las instituciones. Esto puede traer una serie de beneficios para el aprendizaje de los alumnos de segundo año al observar cómo los estudiantes de niveles superiores argumentan o critican los estímulos de discusión. Al respecto, señala González (2012) que Facebook facilita el "sacar al estudiante de su aislamiento y ponerlo en conexión con lo que hacen otros estudiantes, creando relaciones de interdependencia positiva, para pasar de la 'competición' a la 'colaboración', desarrollando habilidades de carácter social para poder trabajar mejor y obtener el máximo rendimiento" (pp. 53, 54).

También interesó indagar a través de la encuesta el posible efecto de inhibición a la participación que podría haber generado el que se premiara a los estudiantes que hubieran elaborado, a juicio de los administradores del grupo, los mejores comentarios de la semana. Ante esto un $36 \%$ de los encuestados 
indica que participaba para profundizar en los contenidos de la asignatura (Motivación intrínseca), un 36\% de alumnos indicó asimismo que participa con el propósito de ganarse la bonificación (Motivación extrínseca). Un $8 \%$ participa para manifestar su opinión y un porcentaje importante (20\%) señala que otra razón lo llevó a participar. Respecto de la influencia que pudo o no tener el hecho de que se decidiera usar esta estrategia de motivación extrínseca (bonificaciones a los mejores comentarios) estudiantes opinaron en el grupo de Facebook: Siendo bastante sincera la motivación de mi primera participación sí fue en el marco de ganar décimas (...), sin embargo después comenzó a interesarme lo que opinaban mis compañeros o a veces los mismos administradores (Estudiante 4) ; ...La primera motivación sinceramente son las décimas pero cuando surge la discusión se vuelve en una buena instancia para aprender más sobre él (Estudiante 2).

Tabla 3: Evaluación de Face-Crític

\begin{tabular}{|l|l|}
\hline Categoría/Reactivo & Frecuencia \% \\
\hline De todos los estímulos me parecieron más interesantes & \\
\hline Videos & $44 \%$ \\
\hline Citas & $23 \%$ \\
\hline Fotografías & $21 \%$ \\
\hline Artículos & $5 \%$ \\
\hline No contesta & $7 \%$ \\
\hline ¿Cuál es el principal uso que hago del grupo? & \\
\hline Leer o mirar lo que se sube & $37 \%$ \\
\hline Ver si hay una información de la asignatura que deba saber & $60 \%$ \\
\hline No contesta & $3 \%$ \\
\hline ¿Qué razones he tenido para participar en el grupo? & \\
\hline Optar a ganarme una bonificación & $36 \%$ \\
\hline Expresar mi opinión & $8 \%$ \\
\hline Profundizar en los contenidos de la asignatura & $36 \%$ \\
\hline Otro & $20 \%$ \\
\hline
\end{tabular}

Estas opiniones muestran que para algunos estudiantes la decisión de ser estimulados a través de un sistema de bonificación junto a las retroalimentaciones a sus comentarios, fue un incentivo inicial a participar. Sin embargo un estudiante criticó esta estrategia de motivación, aun cuando reconoce el aporte al desarrollo del pensamiento crítico, opinando: La idea de este proyecto en sí me parece excelente, noble. Qué más noble que querer generar pensamiento crítico en un estudiante? Supongo que ese es el propósito de un docente; la cuestión es cómo. Cada vez que veo una notificación de este grupo quisiera pensar que es por un sentimiento autónomo y desinteresado, pero creo que lamentablemente cumplió su función a medias. Se ha generado discusión, sí; se ha generado pensamiento crítico, sí; se ha generado una motivación intrínseca para desarrollar el pensamiento crítico, no sé (...) ¿quién no comentaría por unas míseras décimas? "... y es que la discusión no es en sí para generar conocimiento, la discusión es porque tengo que tener un argumento mejor para yo ganarme las décimas. El desafío es, quizás, idear algo similar que busque la generación del pensamiento crítico por desinterés... (Estudiante 5)

La mayoría de los alumnos utilizan Facebook para observar si hay información de la asignatura que el profesor ha comunicado por esa vía. En este sentido se observa la ventaja que tiene el uso de Facebook 
para comunicar información relevante a los estudiantes de manera rápida y eficiente. Al analizar este aspecto en contraposición al uso para el aprendizaje que realizan los estudiantes, (solo un $37 \%$ ) usa la plataforma para leer o mirar lo que se subía a la plataforma. Estos porcentajes nos llevan a pensar respecto de la orientación de los estudiantes sobre el proceso de aprendizaje y la valoración que realizan a aspectos administrativos o formales de la asignatura. En base a estas consideraciones, se nos plantea la dificultad que tiene el uso de estas herramientas virtuales para evaluar la participación de los alumnos, ya que solo podemos observar la participación activa (o comunicación dirigida) como la escritura de comentarios, o "me gusta". Sin embargo como ya hemos comentado, también existen otras formas de participación, por ejemplo alumnos que solo leen o miran lo que otros publican, comentan o comparten.

\section{CONCLUSIONES}

A partir del análisis precedente podemos concluir que: 1) Los alumnos manifiestan una actitud positiva al uso de este grupo en Facebook como complemento a las actividades en el aula. 2) Face-Crític funcionó como un espacio extra aula para profundizar en los distintos enfoques, metodologías y supuestos que están a la base de la psicología de la personalidad. 3) Según la percepción de los alumnos, Face-Crític funciona como un espacio propicio para el desarrollo de la competencia transversal "Pensamiento crítico" en la formación de psicólogos. 4) Es necesario considerar la estrategia motivacional empleada y un método adecuado que permita conocer con más precisión los patrones de participación que los alumnos realizan de esta plataforma 5) Una nueva implementación de Face-Crític en otros contextos podrá considerar los beneficios y limitaciones de su uso como metodología de aprendizaje detectados en esta experiencia.

\section{AGRADECIMIENTOS}

Este trabajo se enmarca en el Proyecto "Face-Crític: Favoreciendo el pensamiento crítico y el aprendizaje autónomo a través de un espacio virtual" financiado por la Unidad de Mejoramiento Docente perteneciente a la Dirección de Docencia de la Universidad de La Serena a través del Concurso Buenas Prácticas Docentes 2014.

\section{REFERENCIAS}

Abarca, S., Las Redes Sociales como Instrumento de Mediación Pedagógica: Alcances y Limitaciones, http://www.redalyc.org/articulo.oa?id=44727049013, ISSN 1409-4703, Revista Electrónica Actualidades Investigativas en Educación, 13(2) 1-18 (2013)

Aguirre, A. y N. Manasía, Web 2.0 y Web Semántica en los Entornos Virtuales de Aprendizaje, http://redalyc.uaemex.mx/src/inicio/ArtPdfRed.jsp?iCve=90412325012, ISSN (Versión impresa): 13172255, 9(3), 320-328 (2009)

Álvarez, F., J.R. Rodríguez, E. Sanz y M.M. Fernández, Aprender Enseñando: Elaboración de Materiales Didácticos que facilitan el Aprendizaje Autónomo, http://dx.doi.org/10.4067/S0718-50062008000600004, Formación Universitaria, 1 (6), 19-28 (2008)

Betancourth, S., K. Insuasti y N. Riascos, Pensamiento Crítico a través de la Discusión Socrática en Estudiantes Universitarios, http://www.redalyc.org/articulo.oa?id=194224362009, ISSN: 0124-5821, Revista Virtual Universidad Católica del Norte, 35,147-167 (2012)

Bosch, T. Using Online Social Networking for Teaching and Learning: Facebook use at The University of Cape Town, DOI:10.1080/02500160903250648, Communicatio: South African Journal for Communication Theory and Research, 35(2), 185-200 (2009)

Bravo, M. Enseñanza-aprendizaje de las Matemáticas utilizando como apoyo Ambientes Virtuales de Aprendizaje, en Las Tecnologías de la Información en Contextos Educativos: Nuevos Escenarios de Aprendizaje, Editorial Universidad Santiago de Cali, pp. 177-202, Santiago de Cali, Colombia, (2012)

Burke, M., C. Marlow y T. Lento, Social Network Activity and Social Well-being, http://dx.doi.org/10.1145/1753326.1753613,Postgraduate Medical Journal, 85, 455-459 (2010)

Cabrera, P., A. Larraín, R. Moretti, M. Arteaga, y M. Energici, La Formación en Psicología desde una perspectiva de Competencias. Una Contribución para el Mejoramiento de la Formación Universitaria en Chile, http://www.cned.cl/public/Secciones/SeccionRevistaCalidad/doc/68/cse_articulo961.pdf, ISSN-e 0717-4004, Calidad en la Educación, 33, 183-223 (2010) 
Çelik, L., Evaluation of the views of Pre-Service teachers taught with Moodle during the course named "Instructional Technology and Material Design" on the Use of Teaching Materials, DOI: 10.1016/j.sbspro.2010.12.402, ProcediaSocial and Behavioral Sciences, 9, 1793-1797 (2010)

Colegio de Psicólogos de Chile. Código de Ética Profesional, 1-36, Colegio de Psicólogos de Chile, Santiago, Chile, (1999)

Davies, M., Introduction to the Special Issue on Critical Thinking in Higher Education, http://dx.doi.org/10.1080/07294360.2011.562145, Studies in Higher Education, 30 (3), 255-260 (2011)

Domenech, M. M., J.A. Erickson, J.T. Thomas, L. Forrest, A.J. Anderson, J.N. y Bow, . Ethics Education in Professional Psychology: A Survey of American Psychological Association Accredited Programs. http://dx.doi.org/10.1037/tep0000043, Training and Education in Professional Psychology, 8, 241-247 (2014)

Fernández, E., y A. Bernardo, Autoeficacia en la Autorregulación del Aprendizaje de Estudiantes Universitarios, http://infad.eu/RevistalNFAD/2011/n1/volumen3/INFAD_010323_201-208.pdf, ISSN: 021498, International Journal of Developmental and Educational Psychology, 1 (3), 201-208 (2011)

González, E., M.D. De Juan, J.F. Parra, F. J. Sarabia Sánchez y A. Kanther, Aprendizaje Autorregulado: Antecedentes y Aplicación a La Docencia Universitaria de Marketing, Revista de Investigación Educativa, 28(1), 171-194 (2010)

González, M. Uso de Blogs y Redes Sociales para el Aprendizaje de Lenguas Extranjeras en un Contexto Universitario, Núcleo, 24(29) 39 - 57 (2012)

González, M.I., I.N. González y K.D. Vicencio, Descripción del Rol Autopercibido del Psicólogo y sus Implicancias en los Procesos de Formación de Pregrado, http://dx.doi.org/10.5027/psicoperspectivasVol13-Issue1-fulltext-296, Psicoperspectivas,13(1), 108-120 (2014)

Hammer, L y C.J. Noemi, Relación entre Pensamiento Crítico y Complejidad Discursiva en Estudiantes Universitarios. doi 10.7764/onomazein.32.10, Onomazein, (En prensa)

Hample, D., Arguing: Exchanging Reasons Face to Face. Mahwah, NJ: Erlbaum (2005)

Hample, D., B. Han, y D. Payne, The Aggressiveness of Playful Arguments. DOI 10.1007/s10503-0099173-8, Argumentation 24: 405-421 (2010)

Inzunza, B.C., R.A. Rocha, C.G. Márquez y M.S. Duk, Asignatura Virtual como Herramienta de Apoyo en la Enseñanza Universitaria de Ciencias Básicas: Implementación y Satisfacción de los Estudiantes, doi: 10.4067/S0718-50062012000400002, Formación Universitaria, 5(4), 3-14 (2012)

Jia, J., Y. Chen, Z. Ding, y M. Ruan, Effects of a Vocabulary Acquisition and Assessment System on Students' Performance in a Blended Learning Class for English Subject, Computers and Education 58, 6376 (2012)

Lamas, H., Aprendizaje Autorregulado, Motivación y Rendimiento Académico, http://www.redalyc.org/articulo.oa?id=68601402, ISSN: 1729-4827, Liberabit. Revista de Psicología, 14, $15-20(2008)$

Machado, A.M. Exercising Critical Stance: Challenges in a School Psychology Internship, http://dx.doi.org/10.1590/1982-3703001112013, Psicologia: Ciência e Profissão, 34(3), 761-773 (2014)

Manterola, M. Los Procesos Mentales y el Aprendizaje: El Enfoque Cognoscitivo. En Psicología educativa: conexiones con la sala de clases, 2a edición, por M. Manterola, pp 127-21 Universidad Católica Blas Cañas, Santiago, Chile (2003)

Micheli, J. y S. Armendáriz, Estructuras de Educación Virtual en la Organización Universitaria: Un Acercamiento a la Sociedad del Conocimiento, http://dx.doi.org/10.4067/S0718-50062011000600005, Formación Universitaria, 4(6), 35-48 (2011)

Moore, T., Critical Thinking: Seven Definitions in Search of a Concept, http://dx.doi.org/ 10.1080/03075079.2011.586995, Studies in Higher Education, 38 (4), 506-522 (2013) 
Moreno, M.J. y R.D. Quiñones, La Perspectiva Didáctica de la Estimulación Motivacional en el Proceso de Enseñanza-Aprendizaje, Revista Iberoamericana Educación, 48(4):1-12 (2009)

Muñoz-Cano, J. M., J.A. Córdiva y H. Priego, Dificultades y Facilidades para el Desarrollo de un Proceso de Innovación Educativa con Base en Las Tecnologías de la Información y Comunicación, Formación Universitaria, 5(1), 3-12 (2012)

Nokes, J. D., J.A. Dole y D. J.Hacker, Teaching High School Students to use Heuristics while Reading Historical Texts, http://dx.doi.org/10.1037/0022-0663.99.3.492.supp, Journal of Educational Psychology. 99, 492-504 (2007)

Paragina, F., S. Paragina, A. Jipa, T. Savu, Dumitrescu, A., The Benefits of Using MOODLE in teacher training in Romania, DOI: 10.1016/j.sbspro.2011.03.252, Procedia Social and Behavioral Sciences, 15, 1135-1139 (2011)

Paul, R. y L. Elder, Una Guía para los Educadores en los Estándares de Competencia para el Pensamiento Crítico. Estándares, Principios, Desempeños y Resultados. (2005). https://www.criticalthinking.org/resources/PDF/SP-Comp_Standards.pdf Acceso: 12 de junio de 2015

Peñalosa, C., P. Landa, y C.Z. Vega, Aprendizaje Autorregulado: Una Revisión conceptual. http://www.revistas.unam.mx/index.php/repi/article/viewFile/19017/18042, Revista Electrónica de Psicología Iztacala, 9 (2), 1-21 (2006)

Red Psicología Proyecto MECESUP ULS 0601. Competencias del perfil de egreso de psicólogo. En M. T. Juliá (Ed.), Competencias del Psicólogo en Chile. Propuestas desde Las Universidades Estatales, pp. 166 -188, La Serena: Editorial Universidad de La Serena (2013)

Rodríguez-Ponce, E. y A. Palma-Quiroz, Desafíos de la Educación Superior en la Economía del Conocimiento, http://dx.doi.org/10.4067/S0718-33052010000100002, Ingeniare. Revista chilena de ingeniería, 18(1), 8-14 (2010)

Sánchez, M., y otros 5 autores, Entorno Virtual de Enseñanza-Aprendizaje para la Construcción del Conocimiento en Bioquímica Médica. http://www.scielo.org.ve/scielo.php?pid=S079804692009000100006\&script=sci_arttext, ISSN 0798-0469, 32 (1), 31-37 (2009)

Santibañez, C. ¿Para qué sirve Argumentar?: Problematizando Teórica y Empíricamente el Valor y la Función de la Argumentación, http://dx.doi.org/10.5209/rev_CLAC.2014.v58.45474, Círculo de lingüística aplicada a la comunicación, 58, 163-205 (2014)

Sava, S., L. Malita y E. Nuissl, Quality of the Online Delivery in the European Master in Adult Education, doi:10.1016/j.sbspro.2010.12.385, Procedia - Social and Behavioral Sciences, 9, 1687-1691 (2010)

Shaw, A. M. K.R. Timpano, Tran, T. B. y Joormann, J. Correlates of Facebook Usage Patterns: The Relationship between Passive Facebook Use, Social Anxiety Symptoms, and Brooding, http://dx.doi.org/10.1016/j.chb.2015.02.003, Computers in Human Behavior, 48, 575-580 (2015)

Universidad de La Serena. Modelo Educativo (en línea). (2011) Recuperado el 25 de abril del 2014 de http://www.userena.cl/archivos/modelo_educativo_ULS.pdf

Wass, R., T. Harland y A. Mercer, Scaffolding Critical Thinking in the Zone of Proximal Development, http://dx.doi.org/10.1080/07294360.2010.489237, Studies in Higher Education, 30 (3), 317-328 (2011)

Young, A. y C. Norgard, Assessing the Quality of Online Courses from the Students' Perspective, http://dx.doi.org/10.1016/j.iheduc.2006.03.001, The Internet and Higher Education, 9 (2), 107-115 (2006)

Zuñiga, C. Diagnóstico de Necesidades de Formación en Psicología a partir del Discurso de diversos Actores Sociales. En M. T. Juliá (Ed.), Competencias del Psicólogo en Chile. Propuestas desde Las Universidades Estatales, pp. 83-98, La Serena: Editorial Universidad de La Serena (2013) 
